\title{
Educação continuada em enfermagem psiquiátrica: reflexão sobre conceitos
}

\author{
CONTINUING EDUCATION IN PSYCHIATRIC NURSING: \\ A REFLECTION ON CONCEPTS
}

\section{EDUCACIÓN CONTINUA EN ENFERMERÍA PSIQUIÁTRICA: REFLEXIÓN SOBRE CONCEPTOS}

\author{
Maria da Graça Girade ${ }^{1}$, Emirene Maria Navarro Trevizan da Cruz $^{2}$, Maguida Costa Stefanelli ${ }^{3}$
}

\section{RESUMO}

A preocupação com programas para capacitar o enfermeiro, para atuar em enfermagem psiquiátrica, levou-nos a este estudo, o primeiro da linha de pesquisa "Desenvolvimento do pessoal de enfermagem na área da psiquiatria e saúde mental". O objetivo deste foi fazer uma reflexão sobre os conceitos de educação continuada, em serviço e permanente existentes na literatura mais recente sobre o assunto. Para o levantamento bibliográfico dos conceitos em pauta, utilizamos as bases de dados MEDLINE e LILACS, dissertações e teses da área da enfermagem e alguns títulos clássicos sobre o assunto. Após análise dos conceitos e discussão com peritos, concluímos que a denominação educação contínua em serviço é a que guarda mais consonância com os autores e peritos consultados e a que mais atende às demandas de conhecimento existentes na área de assistência de enfermagem psiquiátrica rumo à qualidade da assistência.

\section{DESCRITORES}

Educação continuada. Enfermagem psiquiátrica. Capacitação em serviço.

\section{ABSTRACT}

The concern with continuing education programs that prepare nurses to work in psychiatric nursing led us to conduct this study, the first one on the "development of psychiatric and mental health nursing personnel" research line. Its objective is to make a reflection on the concepts of continuing education, inservice and permanent, found in recent nursing education literature. To carry out the literature review of the concepts we reviewed the MEDLINE and LILACS data bases, theses and dissertations and a number of classical titles on the theme. After the analysis of the concepts and discussions with experts, we concluded that the continuing inservice education denomination is the one that presents more consonance with the authors and experts that were consulted, as well as the one that better meets the demands of knowledge in the area of psychiatric nursing towards the quality of assistance.

\section{KEY WORDS}

Continuing Education.

Psychiatric nursing.

In-service training.

\section{RESUMEN}

La preocupación con programas para capacitar al enfermero, para actuar en enfermería psiquiátrica, nos llevó realizar este estudio, el primero de la línea de investigación "Desarrollo del personal de enfermería en el área de la psiquiatría y salud mental”. El objetivo de este estudio fue hacer una reflexión sobre los conceptos de educación continua, en servicio y permanente existentes en la literatura más reciente sobre el asunto. Para el levantamiento bibliográfico de los conceptos mencionados, utilizamos las bases de datos MEDLINE y LILACS, disertaciones y tesis del área de la enfermería y algunos títulos clásicos sobre el asunto. Después del análisis de los conceptos y discusión con expertos, concluimos que la denominación educación continua en servicio es la que guarda más consonancia con los autores y expertos consultados y la que más atiende a las demandas del conocimiento existentes en el área de la asistencia de enfermería psiquiátrica rumbo a la calidad de la asistencia.

\section{DESCRIPTORES}

Educación contínua.

Enfermería psiquiátrica.

Capacitación en servicio.
1 Enfermeira. Espe-
cialista em Enfer-
magem Psiquiátrica.
Professora Respon-
sável pela Disciplina
Enfermagem Psi-
quiátrica e colabo-
radora na Disciplina
Saúde Mental da
Faculdade de Enfer-
magem de São José
de Rio Preto. Aluna
do Programa de Pós-
graduação (Mes-
trado) em Ciências
da Saúde da Facul-
dade de Medicina de
São José de Rio
Preto (FAMERP).
2 Médica.Professora
Titular do Departa-
mento de Psiquiatria
e Psicologia Médica
da FAMERP.
Orientadora.
3 Enfermeira. Profes-
sora Titular de Enfer-
magem. Orientadora
do Programa de Pós-
Graduação da Escola
de Enfermagem da
USP. Assessora do
Conselho Diretor do
Instituto de Psiquiatria
do Hospital das
Clínicas da Facul-
dade de Medicina da
Universidade de São
Paulo (USP).
mcstefanelli@terracom.br 


\section{INTRODUÇÃO}

A necessidade constante de atualização epistemológica, frente às mudanças tecnológicas de nosso tempo, impõe que se reflita sobre novas estratégias para capacitar o enfermeiro que atua no contexto da assistência psiquiátrica, dada a rapidez e a abrangência do processo evolutivo do conhecimento e dado, outrossim, o papel historicamente reservado à formação do enfermeiro, que na maioria das vezes não privilegia as ações específicas da prática diária da Enfermagem Psiquiátrica, uma vez que o objetivo da graduação é a formação do enfermeiro generalista.

Esse enfermeiro, muitas vezes, vai trabalhar em Hospital Psiquiátrico ou em outros serviços de assistência psiquiátrica e surpreende-se com a sua falta de conhecimento específico, vivendo uma situação de emaranhamento de papéis que dificulta seu ajustamento. Apesar dos seus esforços, pois mesmo sem a formação específica atua nessa área e, em geral, ele não pode considerar-se parte integrante e efetiva da equipe que assiste as pessoas com transtornos mentais, tendo em vista seu frágil conhecimento e o fato de ter de concentrar-se em ações de âmbito burocrático.

Mesmo quando o enfermeiro que atua espontaneamente na área de psiquiatria, sem formação específica ou aquele que desempenha o seu papel, pois são poucos os especialistas, procura cursos de atualização ou especialização, muitas vezes defronta com barreiras como a sobrecarga de trabalho e a falta de apoio da própria instituição, que gera a desmotivação. O enfermeiro necessita manterse atualizado para enfrentar as mudanças advindas do desenvolvimento técnico-científico, devido as novas descobertas em diferentes campos, como os da genética, neuroimagem, ritmo circadiano, neurofisiologia, psicofarmacologia, psico-imunologia, bem como, mudanças nas políticas de saúde mental.

O enfermeiro tem de manter-se em processo de aprendizagem contínua engajando-se em programas de educação continuada, procurando, promovendo ou exigindo da instituição na qual trabalha apoio para à vida profissional na área específica de atuação.

Face a essa realidade, nossa responsabilidade torna-se imensurável como docentes dessa área. No cumprimento de nossa missão, temos que estimular reflexões que propiciem mudanças de atitude para viabilizar o avanço da assistência em enfermagem psiquiátrica e saúde mental.

Temos, portanto, como objetivo do presente trabalho fazer uma reflexão sobre os conceitos de educação continuada, educação em serviço e educação permanente mais utilizados para denominar os programas de atualização do enfermeiro, com foco na assistência de enfermagem psiquiátrica.

\section{METODOLOGIA}

Trata-se de uma reflexão sobre os diversos conceitos de educação continuada, educação em serviço e educação permanente, mais utilizados em enfermagem em geral e em enfermagem psiquiátrica, a partir de um levantamento bibliográfico efetuado nas bases de dados MEDLINE e LILACS. Considerando-se também dissertações e teses da área da enfermagem, privilegiando as mais atuais e acessíveis, sem desprezar outros clássicos sobre o assunto em enfermagem geral e em enfermagem psiquiátrica, bem como publicação da American Nurses Association (ANA) ${ }^{(1)}$.

Do conteúdo destes extraímos os diferentes conceitos citados mais comuns na literatura de Enfermagem, com foco na área de enfermagem psiquiátrica. A reflexão sobre os conceitos encontrados na literatura foi associada aos dados obtidos na consulta a três peritos da área de enfermagem psiquiátrica sobre os conceitos centrais deste estudo. Um desses, docente de enfermagem de uma escola do interior do Estado de São Paulo, com mais de cinco anos de atuação na área específica e dois enfermeiros responsáveis pelo programa de educação continuada de instituições hospitalares.

\section{DESENVOLVIMENTO}

\section{Breve histórico}

Para melhor compreensão dos conceitos em pauta apresentamos um histórico resumido da evolução da educação e educação continuada. Desde 1920, discute-se a idéia de que a educação deve ser por toda vida, portanto, partimos do pressuposto que a idéia não é nova(2).

Na década de 50, o lema da época era de que as pessoas tinham que se ajustar a um mundo novo em mutação, ou seja, que todo conhecimento sofre transformação e é preciso aprender a capacitar-se, ajustar-se as mudanças. No decênio de 60 , os projetos multinacionais incentivaram a educação de adultos. Em 1966, numa Conferência Geral da UNESCO em Paris, definiu-se para educação, objetivos com programa para os próximos anos, considerando prioritária a idéia de educação contínua, como um processo que deve continuar por toda a vida. Já a década de 70, é uma fase com enfoque crítico, que caracterizou-se por uma tomada de consciência de que o homem educa-se a partir da realidade que o cerca e em interação com outros homens, coeduca-se, como sujeito transformador ${ }^{(2)}$.

O I Seminário de Educação Continuada em Enfermagem, promovido pela Sociedade Brasileira de Enfermagem (ABEn), ainda no primeiro ano da década de oitenta, tornou imperiosa a presença da educação continuada nesta área, visto que aquela "significa a aquisição progressiva de competências, 
que devem ser visíveis na qualidade do exercício da assistência de enfermagem"(3).

Em 1997, foi criada a SOBRECEN - Sociedade Brasileira de Educação Continuada em Enfermagem, que tem norteado o intercâmbio de experiências, nas práticas da educação continuada em enfermagem nas instituições, por meio de reuniões científicas e publicações ${ }^{(2)}$.

Pelo exposto nesse sucinto resumo histórico, temos um aspecto central nos indicando que a aprendizagem é por toda vida, e deve respaldar-se nos princípios de educação de adultos, que é a população para a qual os programas de educação continuada ou contínua em enfermagem são direcionados.

\section{Apresentação de conceitos}

A primeira questão a ser considerada é a abordagem com relação aos conceitos sobre educação continuada, educação em serviço e educação permanente com vistas ao aprimoramento profissional. Essas são as expressões mais utilizadas na literatura da área da saúde para abordar o presente tema, por vezes de forma complementar, outras de modo impreciso ou como conceitos distintos.

Educação continuada é definida pela Organización Panamericana de Salud como um processo permanente de educação, complementando a formação básica, objetivando atualização e melhor capacitação de pessoas e grupos, frente as mudanças técnico científicas ${ }^{(4)}$.

Para a Organización Mundial de la Salud, a educação continuada consiste no processo que inclui todas as experiências posteriores a formação inicial ${ }^{(5)}$.

De acordo com as definições da $\mathrm{ANA}^{(1)}$ sobre educação continuada e desenvolvimento de pessoal, conclui-se que ambas se complementam nos aspectos de orientação e de educação em serviço, com a finalidade de desenvolver o indivíduo integralmente, como profissional e como pessoa de acordo com os objetivos e responsabilidades da instituição. Para esta entidade, educação continuada estende-se por toda a vida profissional e contribui para o desenvolvimento do enfermeiro.

Educação continuada é aquela que capacita a pessoa não só para as mudanças desejadas pela instituição como também para as requeridas pela sociedade, desenvolvendoa como pessoa e como profissional ${ }^{(6)}$. Alude à atualização técnico-científica do profissional, voltada para a prática individual e é da responsabilidade do profissional, portanto um processo desvinculado da instituição onde atua o profissional, feita de modo esporádico, com apropriação do saber científico ${ }^{(7)}$.

Especificamente em relação à área de Enfermagem, este tipo de educação constitui-se em veículo para que os enfer- meiros respondam não só às rápidas mudanças no conhecimento aperfeiçoando seu desempenho no cuidado à saúde, mas também contribuindo para a elevação dos padrões profissionais de sua prática corrente. Esta última está relacionada ao atendimento das exigências da instituição ${ }^{(8)}$.

Vê-se, nessa rápida viagem pela literatura, que os elementos nucleares usados para a definição de educação continuada ou em serviço não são constantes.

Educação permanente é outra expressão bastante utilizada na área de Saúde. Esta não é recente, pois o filósofo chinês Lao-Tsé dizia há sete séculos A.C. que todo estudo é interminável ${ }^{(9)}$.

A Organização das Nações Unidas para a Educação, Ciências e Cultura (UNESCO) na década de setenta, conceituou educação permanente a partir do princípio de que o homem se educa à vida inteira, atentando para o seu desenvolvimento pessoal e profissional, a evolução das capacidades, motivações e aspirações e que as suas necessidades nem sempre são de caráter emergente ${ }^{(10)}$.

A educação permanente pode ser definida como um processo de ensino e aprendizagem dinâmico e contínuo, tendo como finalidade a análise e aprimoramento da capacitação de pessoas e grupos, para enfrentarem a evolução tecnológica, as necessidades sociais e atenderem aos objetivos e metas da instituição a que pertencem ${ }^{(11-12)}$.

A educação permanente é também apresentada como prática institucionalizada, com o objetivo de promover mudança institucional, fortalecer as ações de equipe, transformar práticas técnicas e sociais, adotando-se, para tanto, uma pedagogia centrada na resolução de problemas e efetuada no ambiente de trabalho, de maneira a promover a apropriação do saber científico, configurando-se como responsabilidade da instituição na qual o profissional de saúde atua ${ }^{(7)}$.

Educação em serviço é uma unidade desenvolvida por meio de programas, que orienta os profissionais, de acordo com os objetivos da instituição ${ }^{(13)}$. Esse conceito guarda semelhança com o anterior que refere-se a educação permanente. Não há, portanto, consenso entre os autores.

Para ANA, educação em serviço refere-se às atividades no ambiente de trabalho desenvolvidas para que o profissional possa adquirir, manter e aumentar sua competência, visando o cumprimento de suas responsabilidades ${ }^{(1)}$. Outro autor a considera como a capacitação da pessoa para o trabalho específico, portanto, como um tipo de educação cujo desenvolvimento processa-se no ambiente de trabalho, voltada para uma instituição em particular ${ }^{(6)}$.

Como vimos, há diversas maneiras de conceituar educação continuada, educação permanente e em serviço, mas todas tem propósitos definidos que culminam, de forma integrada ou não, com o de atendimento das metas da institui- 
ção, desenvolvimento profissional e pessoal, acréscimo de conhecimentos, aprimoramento de habilidades e promoção de mudanças de atitudes.

Não podemos deixar de considerar que seja qual for a denominação utilizada temos de ter em mente que trata-se de programas de educação voltados para adultos que tem suas experiências vividas, um repertório de vida, esquema de assimilação, experiências educacional e profissional que exigem mais que um simples artifício pedagógico no processo ensino aprendizado, porquê as características citadas o diferenciam do jovem ${ }^{(14-15)}$.

Discutimos esses conceitos com três peritos nessa área específica. Sugiram algumas discrepâncias quanto as conceituações em estudo, semelhantes às encontradas na literatura, apresentadas porém em menor grau. A tônica comum, implicita ou explicita, foi a contribuição desses programas na aquisição de conhecimentos e habilidades, além da mudança de comportamento em prol da qualidade da assistência.

\section{A educação continuada e a assistência de enfermagem psiquiátrica}

Nas últimas décadas, muito se tem discutido sobre a questão de que a graduação, qualquer que seja a área do conhecimento, não gera profissionais totalmente capacitados para todas as áreas do saber. Essa consideração evidencia que complementações e atualizações futuras tornam-se necessárias e fala a favor da integração de conhecimentos no nível de graduação $^{(16)}$.

Dessa formação, resulta um enfermeiro que não pode assumir o compromisso com a assistência psiquiátrica em seus diferentes níveis de atendimento, pois é exigido dele que conheça todos os serviços que compõem o continuиm desta assistência ${ }^{(17)}$. Esta é uma situação encontrada também em outras especialidades, porque o objetivo da graduação é formar o enfermeiro generalista.É impossível com a celeridade do conhecimento e descobertas científicas formar profissionais com domínio em todas as áreas da enfermagem.

Há publicações na área da Enfermagem que reiteram a necessidade da atualização constante do conhecimento $^{(6,16,18-21)}$. Por sua vez, discute-se a necessidade de educação continuada na área da Saúde e a idéia central é que essa questão deve ser discutida já no mundo acadêmico ${ }^{(22)}$. O que não deixa de ser um estímulo para que o estudante, futuro enfermeiro, assuma a responsabilidade pela sua educação continuada e das futuras ações a respeito do assunto.

Para reforçar essa necessidade, comentamos o achado de um dos autores consultados sobre o fato do conheci- mento adquirido na graduação ter meia-vida de, aproximadamente, dois anos e meio, período após o qual, ele se torna obsoleto, devendo o enfermeiro prosseguir continuamente em processo de atualização ${ }^{(8)}$. Face a evolução e velocidade da disseminação do conhecimento científico, considerando que essa observação foi feita há mais de 25 anos, a meia vida dos cursos de graduação deve ter diminuído sensivelmente.

Com os avanços do conhecimento e as mudanças na assistência psiquiátrica e em saúde mental, o enfermeiro deve renovar seus conhecimentos, repensar suas atitudes e ações que influenciam na busca de mais conhecimentos para assumir com competência a tríade que apóia a qualidade da assistência: educação, pesquisa e prática da enfermagem psiquiátrica $^{(17,23)}$.

\section{CONSIDERAÇÕES FINAIS}

Em virtude do impacto do alto grau de inovação tecnológica alcançado, ao longo de todo o século vinte, e que continua no início do atual, podemos perceber que ocorreram, tanto na vida profissional como nas relações interpessoais de todo ser humano, mudanças significativas na forma como o profissional interage com o conhecimento específico de sua área de atuação, bem como, na forma como as instituições sistematizam o recrutamento e a seleção de profissionais, que sofreu sensível alteração. Essas passaram a exigir um profissional cada vez mais qualificado.

A prática da Enfermagem Psiquiátrica tem exigido, uma maior percepção das necessidades do paciente, e o enfermeiro tem de desenvolver habilidades que lhe possam conferir maior competência para o melhor desempenho de suas funções, com educação contínua (continuing), principalmente no que se refere à cooperação interdisciplinar, com foco no cuidado que visa o atendimento do paciente em todas as suas dimensões, independente do tipo de serviço em que atue.

A educação continuada em serviço para o enfermeiro psiquiátrico é importante para aquisição de habilidades e conhecimentos fundamentais para a qualidade do desempenho de suas ações no cuidado ao paciente, pois "são pessoas cuidando de pessoas" ${ }^{(13)}$.

Infelizmente, dada a escassez de enfermeiros na área psiquiátrica, quem a procura, em geral, é o recém graduado que ao inserir-se no mercado do trabalho, não poucas vezes inicia suas funções na coordenação e gerenciamento, experimentando ansiedade, insegurança e sem preparo, tanto do ponto de vista técnico como do emocional, para assumir funções administrativas, responsabilidade pelo serviço de enfermagem e, ainda, oferecer uma adequada assistência ao paciente ${ }^{(24)}$. 
De acordo com o exposto e a experiência real dos autores na área específica, de vinte a quarenta anos, podemos considerar que boa parte dos enfermeiros tem reavaliado seus conceitos, valores, crenças e visão de mundo em relação à assistência aos portadores de transtornos mentais, com tratamentos diferenciados, procurando o aprimoramento de seus conhecimentos para melhorar a qualidade da assistência por meio de tomada de decisões conscientes que realmente beneficiem o portador de transtorno psiquiátrico, sua família e a sociedade. A nosso ver, o mérito das mudanças provocadas na assistência psiquiátrica foi exigir um repensar de todos os que tem compromisso com essa área, sobre as diferentes formas de prevenção das doenças, de tratamento e cuidado na área da saúde mental.

Esse contexto faz com que os enfermeiros psiquiátricos no nosso meio procurem cursos de especialização ou de aprimoramento em Enfermagem Psiquiátrica e em Saúde Mental, direcionando sua equipe para uma atuação mais participativa, de forma mais efetiva, oferecendo plano terapêutico eficiente - científico e humanitário - à pessoa em sofrimento psíquico ${ }^{(25)}$.

Cabe ressaltar que temos poucos cursos de especialização em Enfermagem Psiquiátrica e Saúde Mental no nosso meio. Os diferentes serviços que oferecem assistência ao portador de distúrbio mental no Brasil precisam de profissionais com conhecimento que possa ser observado na sua produtividade na assistência de enfermagem, e essa produtividade, não pode ser medida numericamente, mas deve ser observada sob a ótica da percepção que o enfermeiro psiquiátrico tem do paciente sob seu cuidado: quanto mais familiarizado o profissional estiver com as técnicas de como lidar, no dia-a-dia, com o paciente psiquiátrico tanto mais se sentirá motivado a prestar uma assistência efetiva e eficaz ao paciente que deverá refletir na adequação de sua prática.

Meios para a aquisição dessas habilidades deverão ser oferecidos nos programas de educação continuada em serviço junto às instituições, que é a forma, por excelência, onde podem ser conciliadas a busca pelo saber especializado e a vida profissional do enfermeiro psiquiátrico.

O verdadeiro processo de educação, só pode ser estabelecido por meio de uma análise das necessidades reais da população envolvida ${ }^{(26)}$. Com as transformações ocorridas nos últimos tempos na psiquiatria com seus avanços científicos, acreditamos ser por esse caminho que os enfermeiros que atuam nessa área ocuparão seu espaço, pois não adianta apenas aumentar a quantidade de conhecimento, mas sim preocupar-se com sua qualidade e a satisfação dos envolvidos no processo - clientela e profissionais. A busca de conhecimento deve ser alicerçada nas reais necessidades de acompanhar a evolução do conhecimento colocando-o à disposição do bem-estar e felicidade do centro do nosso cuidado - o paciente ou cliente.

Para o desenvolvimento pessoal e profissional do enfermeiro que trabalha junto a pacientes com transtornos mentais, a educação contínua em serviço propiciadas pelas instituições apresenta-se como uma alternativa altamente viável para o aprendizado das práticas da Enfermagem Psiquiátrica e em Saúde Mental. Esta deve refletir a filosofia da Instituição que procura a qualidade da assistência vinculada ao conhecimento especializado do enfermeiro psiquiátrico. Para tal, o enfermeiro deve aprofundar-se no conhecimento sobre o processo de comunicação humana e de comunicação terapêutica para desenvolver a relação interpessoal adequada, tão necessária à qualidade do cuidar em Enfermagem Psiquiátrica ${ }^{(27-28)}$. Esta foi destacada recentemente pela Organização Pan-Americana de Saúde, em seu relatório sobre saúde mental ${ }^{29)}$.

Com base no exposto anteriormente, nos autores citados, na discussão com peritos e na nossa vivência consideramos que a educação contínua em serviço, independentemente da denominação que lhe seja atribuída, é o processo de atualização técnico-científico que permite ao profissional a reflexão sobre seus objetivos, sua própria prática, levando-o também ao desenvolvimento pessoal, elevação de sua auto-estima, o que propicia mais gratificação e prazer no seu desempenho profissional. Conseqüentemente, essas mudanças trarão benefícios não só às instituições como, e principalmente, às pessoas assistidas por estes profissionais.

A responsabilidade do enfermeiro pelo seu processo de atualização constante tem de ser estimulada e valorizada. $\mathrm{O}$ ideal seria o desenvolvimento de uma responsabilidade compartilhada entre enfermeiro e instituição.

\section{REFERÊNCIAS}

(1) American Nurses Association (ANA). Council on Continuing Education of Staff Development. Roles and responsabilities for continuing education and staff development across all settings. ANA Publ. 1992;(COE-16 10 M):iii, 1-14.

(2) Ittavo J. Inserção de enfermeiros recém-graduados, admitidos em área hospitalar: um programa de educação conscientizadora [dissertação] Ribeirão Preto: Escola de Enfermagem de Ribeirão Preto/USP; 1997.
(3) Seminário de Educação Continuada em Enfermagem. Rev Bras Enferm. 1980;33(1):98-101.

(4) Organización Mundial de la Salud (OMS). Oficina regional de la organización de la salud. Educación contínua: guia para la organización de programas de educación contínua para el personal de salud. Washington (DC); 1978. 
(5) Organización Mundial de la Salud. Continuando la educación de los trabajadores de salud: principio e guias para el desarrolo de um sistema. Genebra; 1982.

(6) Kurcgant P. Educação continuada: caminho para a qualidade. Rev Paul Enferm. 1993;12(2):66-71.

(7) Almeida MJ. Educação permanente em saúde: um compromisso inadiável. Olho Mágico 1997;5(14):41-7.

(8) McDiarmid S. Continuing nursing education: what resources do bedside nurses use? J Contin Educ Nurs. 1998;20(6): 267-73.

(9) Bagnato MH. Educação continuada na área da saúde: uma aproximação crítica. Campinas: Alínea; 1999.

(10) Delors J. Educação: um tesouro a descobrir. São Paulo: Cortez/ MEC/UNESCO; 1999.

(11) Silva LAA, Saupe R. Proposta de um modelo andragógico de educação continuada para a enfermagem. Texto Contexto Enferm. 2000;9(2):478-84.

(12) Freire P. Educação e mudança. Rio de Janeiro: Paz e Terra; 1999.

(13) Nuñez RS; Luckesi MAV. Educação em serviço: fator de desenvolvimento de recursos humanos em enfermagem. Rev Bras Enferm. 1980;33(1):54-80.

(14) Kurcgant P. Necessidades sentidas e evidenciadas por enfermeiras e obstetrizes do município de São Paulo [dissertação].São Paulo: Escola de Enfermagem da USP;1978.

(15) Silva MJP, Pereira L, Benko MA. Educação continuada: estratégia para o desenvolvimento do pessoal de enfermagem. São Paulo: Marques Saraiva; 1989.

(16) Stefanelli MC, Rolim MA, Teixeira MB, Barros S, Fukuda IMK, Forcella HT, et al. Integração dos conceitos de saúde mental nos cursos de graduação em enfermagem. Rev Paul Enferm. 1996;15(1/3):51-65.

(17) Stefanelli MC. Perspectivas da enfermagem psiquiátrica no século XXI. [Apresentado à Reunião Clínica do Instituto de Psiquiatria do Hospital das Clínicas da Faculdade de Medicina da Universidade de São Paulo; 2003 set. 9; São Paulo].
(18) Leite MMJ, Pereira LL. Educação continuada em enfermagem. In: Kurcgant P. Administração em enfermagem. São Paulo: EPU; 1991. p. 147-63.

(19) Pereira FBG. Pedagogia problematizadora na educação continuada no Hospital das Clínicas -UNICAMP. [dissertação ] Ribeirão Preto: Escola de Enfermagem de Ribeirão Preto/USP; 1996.

(20) Lorencetti DAC. A importância e proposta de indicadores para avaliação dos serviços de educação continuada - segundo gerentes dos serviços de enfermagem. [dissertação] São Paulo: Escola de Enfermagem da USP; 2003.

(21) Bezerra ALQ O contexto da educação continuada em enfermagem. São Paulo: Lemar e Martinale; 2003.

(22) L'Abbate S. Educação e serviços de saúde: avaliando a capacitação dos profissionais. Cad Saúde Pública. 1999;15(supl.2):15-27.

(23) Stuart GW, Laraia MT. Enfermagem psiquiátrica: princípios e prática. $6^{\text {a }}$ ed. Porto Alegre: Artmed; 2001.

(24) Belei RA. Primeiro emprego: como treinar o enfermeiro recém -graduado. Rev Bras Enferm. 1992;45(4):308-12.

(25) Tavares RR. O enfermeiro em diferentes unidades psiquiátricas: educação continuada e propagação do conhecimento [dissertação ] Ribeirão Preto: Escola de Enfermagem de Ribeirão Preto/USP; 2003.

(26) Freire P. Pedagogia da autonomia: saberes necessários à prática educativa. Rio de Janeiro: :Paz e Terra; 2000.

(27) Stefanelli MC. Comunicação com o paciente: teoria e ensino. $2^{\text {a }}$ ed. São Paulo: Robe; 1993.

(28) Arantes ECA. Ensino de relacionamento terapêutico em enfermagem psiquiátrica. Rev Bras Enferm. 1973;26(6):438-46.

(29) Organização Pan-americana da Saúde (OPAS), Organização Mundial da Saúde (OMS). Relatório sobre a saúde no mundo 2001: saúde mental: nova concepção, nova esperança. Genebra: OPAS/OMS; 2001. 